03,04,07

\title{
Сжимаемость и электронные свойства цианидов металлов
}

\author{
(ㄱ Д.В. Корабельников, И.А. Федоров, Ю.Н. Журавлев \\ Кемеровский государственный университет, \\ Кемерово, Россия \\ E-mail: dkorabelnikov@yandex.ru
}

Поступила в Редакцию 3 марта 2021 г.

В окончательной редакции 3 марта 2021 г.

Принята к публикации 8 марта 2021 г.

\begin{abstract}
Сжимаемость и электронные свойства цианидов металлов исследованы на основе теории функционала плотности с учетом дисперсионного взаимодействия Ван-дер-Ваальса. Показано, что цианид золота имеет низкую линейную сжимаемость (менее $0.1 \%$ при давлении $1 \mathrm{GPa})$ и высокий линейный модуль $(\sim 1200 \mathrm{GPa})$ вдоль цепочек $-\mathrm{Au}-\mathrm{CN}-\mathrm{Au}-\mathrm{CN}-$. Цианид серебра демонстрирует отрицательную линейную сжимаемость, которая коррелирует со сжимаемостью координационных связей $\mathrm{Ag}-\mathrm{N}$. Для цианида натрия линейная сжимаемость вдоль ковалентных связей $\mathrm{C}-\mathrm{N}$ больше, чем для цианидов золота и серебра, тогда как упругая анизотропия меньше. В отличие от цианида натрия, для цианидов золота и серебра катион-анионные связи $(\mathrm{Au}-\mathrm{N}, \mathrm{Au}-\mathrm{C}$ и $\mathrm{Ag}-\mathrm{N}, \mathrm{Ag}-\mathrm{C})$ имеют частично ковалентную природу, а верхние валентные состояния отвечают преимущественно состояниям катионов. Ширина запрещенной зоны цианида золота меньше, чем для цианидов серебра и натрия. Ширины запрещенных зон цианидов золота и серебра существенно уменьшаются с ростом давления, что указывает на возможность металлизации при достаточно высоких давлениях.
\end{abstract}

Ключевые слова: цианиды металлов, сжимаемость, давление, ширина запрещенной зоны, теория функционала плотности.

DOI: 10.21883/FTT.2021.07.51036.044

\section{1. Введение}

Цианиды металлов применяют для извлечения золота и серебра из руд, для повышения твердости и износоустойчивости стали. Кроме того, цианиды металлов находят применение в электрохимии (в составе электролитов для гальванического покрытия деталей напылением из золота и серебра) и в органическом синтезе.

Цианиды металлов являются ионно-молекулярными соединениями с молекулярными цианид-анионами $\mathrm{CN}^{-}$ и катионами металлов $\mathrm{M}^{+}$. При нормальных условиях $\mathrm{AgCN}$ кристаллизуется в тригональную структуру (пространственная группа $R 3 m$ ), тогда как $\mathrm{AuCN}$ является гексагональным кристаллом с пространственной группой $P 6 \mathrm{~mm}$ [1]. Цианиды серебра и золота имеют полимерную цепочечную структуру, сформированную линейными цепями $-\mathrm{Ag}-\mathrm{CN}-\mathrm{Ag}-\mathrm{CN}-\left(-(\mathrm{Ag}-\mathrm{CN})-{ }_{n}\right)$ и $-\mathrm{Au}-\mathrm{CN}-\mathrm{Au}-\mathrm{CN}-\left(-(\mathrm{Au}-\mathrm{CN})-{ }_{n}\right)$, соответственно (рис. 1). Цианид натрия при нормальных условиях имеет кубическую структуру, тогда как его низкотемпературная фаза (при $T<180 \mathrm{~K}$ ) относится к ромбической системе (группа Pmmn) [2]. Элементарная ячейка $\mathrm{NaCN}$ содержит две формульные единицы (рис. 1).

Влияние давления на структуру $\mathrm{AgCN}, \mathrm{AuCN}$ и ромбической фазы $\mathrm{NaCN}$ не изучено. Цианиды серебра и золота проявляют отрицательное тепловое расширение (OTP) вдоль цепочек $-(\mathrm{M}-\mathrm{CN}){ }_{n}$ [3]. Материалы с ОТР весьма привлекательны для применения в воло- конной оптике, электронике и различных покрытиях с заданным коэффициентом теплового расширения [4-6]. Также ОТР материалы имеют потенциал для дизайна термомеханических приводов [7]. Как известно, тепловое расширение обусловлено ангармонизмом колебаний (характеризуется параметрами Грюнайзена), вызывающим тепловое давление, а также упругим откликом решетки на это тепловое давление [8]. В ряде случаев тела наряду с ОТР проявляют отрицательную линейную сжимаемость (ОЛС) [9-11]. Возможны корреляции между упругими и тепловыми свойствами, в частности между ОТР и ОЛС [12-14]. Таким образом, цианиды являются перспективными объектами для поиска у них аномальных механических свойств. ОЛС-материалы являются редкими, а их поиск и исследование в последние годы активно ведутся [15-22]. Интерес представляет предсказание возможности аномальной линейной сжимаемости в цианидах. Ковалентные кристаллы, как правило, обладают низкой сжимаемостью и высокой твердостью [23]. Так, объемные модули алмаза и кубического нитрида бора (BN) составляют 443 и $369 \mathrm{GPa}$, соответственно [23]. Различные аллотропные модификации нитрида углерода $\left(\mathrm{C}_{3} \mathrm{~N}_{4}\right)$ также имеют объемные модули выше $320 \mathrm{GPa}[23,24]$. Таким образом, можно предположить, что сжимаемость цианидов металлов в направлении ковалентных связей $\mathrm{C}-\mathrm{N}$ будет мала. При этом интересна роль и природа связей $\mathrm{M}-\mathrm{N}$ и $\mathrm{M}-\mathrm{C}$ в цепочках $-(\mathrm{M}-\mathrm{CN})-{ }_{n}$. Экспериментальные исследования цианида золота указывают на существенное уменьшение 

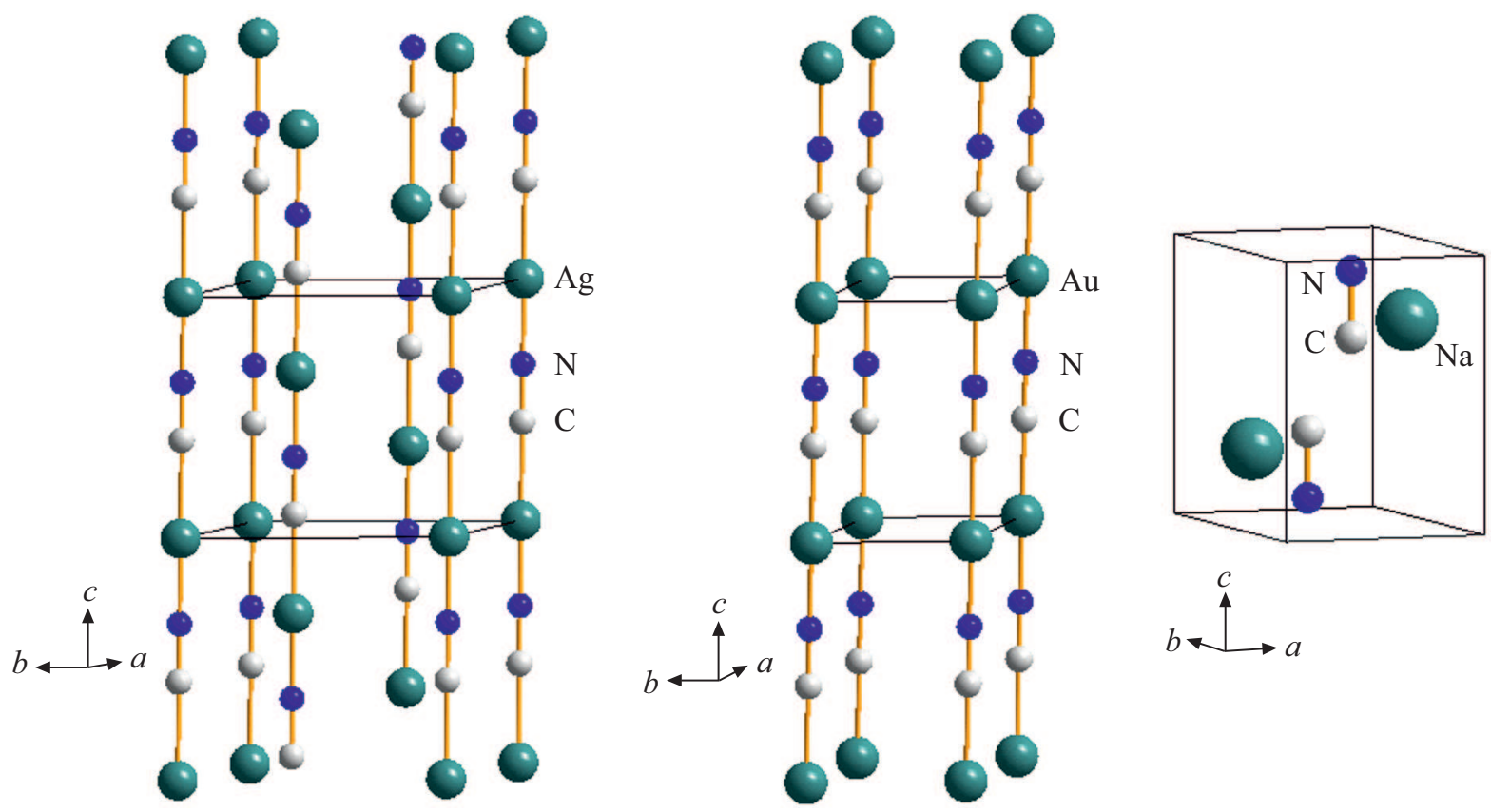

Рис. 1. Структура цианидов $\mathrm{AgCN}, \mathrm{AuCN}$ и $\mathrm{NaCN}$.

его ширины запрещенной зоны с ростом давления и металлизацию при $10 \mathrm{GPa}$ [25]. Влияние давления на ширину запрещенной зоны цианидов серебра и натрия не изучено. Топологический анализ электронной плотности $\mathrm{MCN}$ не проводился.

Расчеты, основанные на теории функционала плотности (DFT), позволяют моделировать кристаллическую структуру и свойства твердых тел (в том числе в условиях внешнего давления) в хорошем согласии с экспериментальными данными [26-33]. Более того, компьютерное моделирование применяется для прогнозирования и дизайна новых материалов [34,35]. Целью настоящей работы является исследование сжимаемости и электронных свойств цианидов металлов с помощью DFT расчетов.

\section{2. Метод расчета}

В настоящей работе DFT-расчеты кристаллической структуры и электронных свойств выполнены с помощью программы CRYSTAL [36]. В вычислениях использовались базисные наборы атомных орбиталей [37-40] и обменно-корреляционный функционал $\mathrm{PBE}$ [41]. Для расчетов ширины запрещенной зоны также применялся гибридный функционал B3LYP [42]. Дисперсионная поправка Гримма DFT-D3(BJ) [43, 44] использовалась для того, чтобы учесть дисперсионные взаимодействия. Первопринципное определение кристаллической структуры было проведено путем минимизации полной энергии, межатомных сил и смещений. Допускалась релаксация всех атомов и параметров решетки согласно алгоритму Broyden-Fletcher-Goldfarb-Shanno (BFGS) [45]. Сходи- мость по энергии была лучше чем $10^{-7} \mathrm{eV}$. Зависимости от давления рассчитывались согласно работе [46]. Химическая связь изучалась на основе квантовой теории атомов в молекулах (QTAIM) Бейдера [47]. Энергии межатомных взаимодействий оценивались с использованием плотности потенциальной энергии в критических точках связи [48]. Код TOPOND [49] использовался для QTAIM топологического анализа электронной плотности.

\section{3. Результаты и их обсуждение}

В табл. 1 для цианидов металлов при нормальном давлении приведены рассчитанные структурные параметры в сравнении с экспериментальными данными $[1,2]$. Вычисленные величины постоянных решетки $a, b, c$ от-

Таблица 1. Рассчитанные и экспериментальные параметры элементарной ячейки для цианидов металлов $\mathrm{MCN}$ $(\mathrm{M}=\mathrm{Ag}, \mathrm{Au}, \mathrm{Na})$

\begin{tabular}{c|c|c|c|c}
\hline Цианид & Метод & $a, \AA$ & $b, \AA$ & $c, \AA$ \\
\hline \multirow{2}{*}{$\mathrm{AgCN}$} & Расчет & 5.947 & 5.947 & 5.348 \\
\cline { 2 - 5 } & эксп. [1] & 5.996 & 5.996 & 5.259 \\
\hline \multirow{2}{*}{$\mathrm{AuCN}$} & Расчет & 3.378 & 3.378 & 5.147 \\
\cline { 2 - 5 } & эксп. [1] & 3.396 & 3.396 & 5.092 \\
\hline \multirow{2}{*}{$\mathrm{NaCN}$} & Расчет & 3.613 & 5.475 & 4.849 \\
\cline { 2 - 5 } & эксп. [2] & 3.630 & 5.450 & 4.850
\end{tabular}



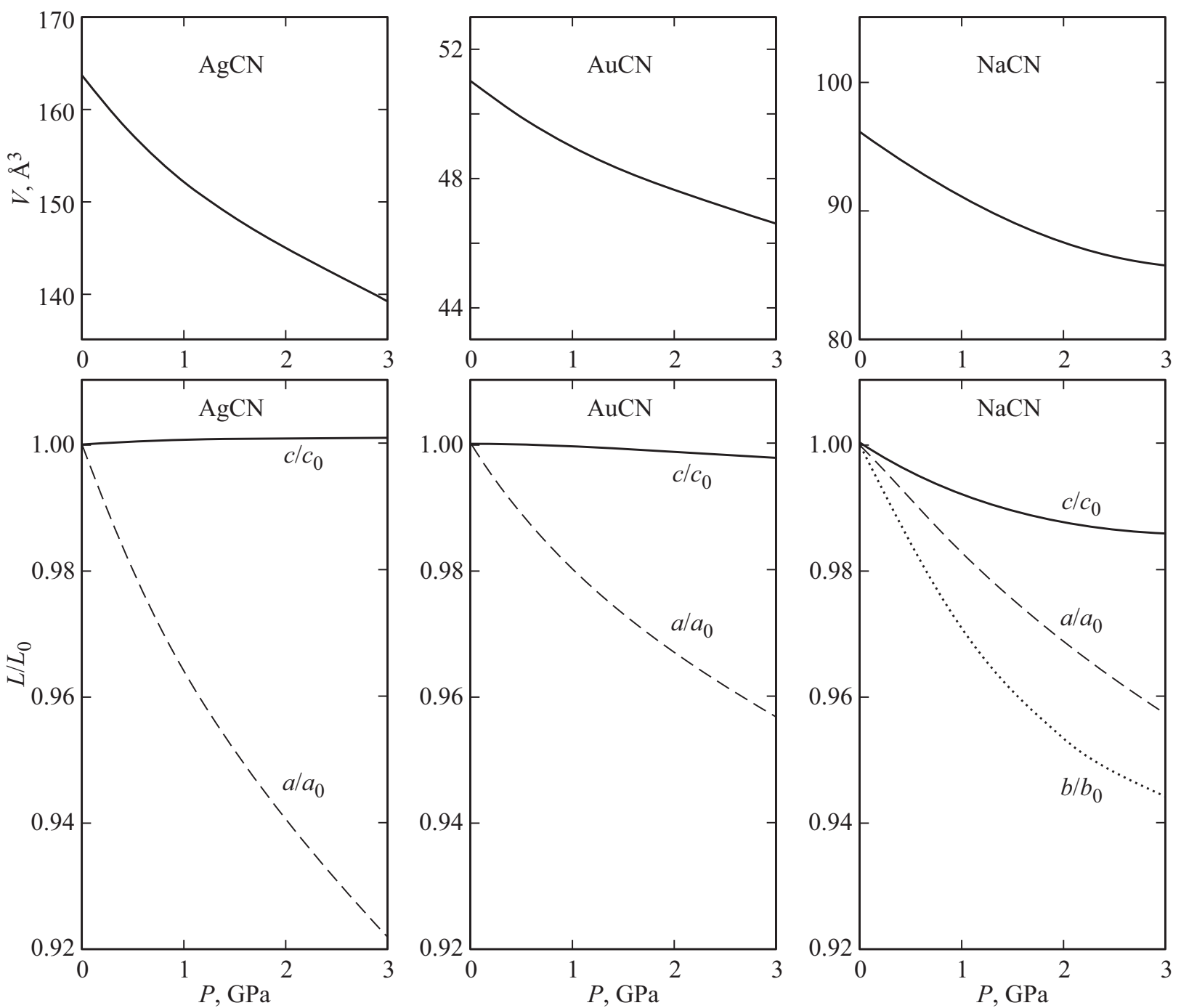

Рис. 2. Рассчитанные зависимости от давления объема $V$ и относительных параметров решетки $a / a_{0}$ (штриховые), $b / b_{0}$ (пунктирные), $c / c_{0}$ (сплошные) для цианидов металлов.

личаются от их экспериментальных значений менее чем на $2 \%$.

На рис. 2 показаны рассчитанные зависимости от давления параметров решетки и объема для цианидов серебра, золота и натрия.

Цианид серебра имеет наибольший объем (на формульную единицу) и наименьший модуль объемного сжатия $11.1 \mathrm{GPa}$. Объемный модуль цианида золота $20.1 \mathrm{GPa}$ больше чем для цианида натрия $(14.3 \mathrm{GPa})$, что может быть обусловлено различием природы межатомных взаимодействий. Из рис. 2 видно, что линейная сжимаемость цианидов металлов сильно анизотропна, в особенности для $\mathrm{AgCN}$ и $\mathrm{AuCN}$. Так, давление $1 \mathrm{GPa}$ вызывает сжатие цианида золота вдоль цепочек $-\mathrm{Au}-\mathrm{CN}-\mathrm{Au}-\mathrm{CN}-$ (вдоль оси $c$ ) менее чем $0.1 \%$. Соответствующий линейный модуль для $\mathrm{AuCN}$ составляет $\sim 1200 \mathrm{GPa}$ и сопоставим с линейными модулями алмаза и нитрида бора [23]. С другой стороны сжимаемость AuCN вдоль осей $a$ и $b$ на порядок больше $(\sim 2 \%$ при $P=1 \mathrm{GPa})$. С ростом давления параметр $c$ цианида серебра увеличивается (рис. 2). Таким образом, давление вызывает увеличение линейных размеров $\mathrm{AgCN}$ вдоль цепочек $-\mathrm{Ag}-\mathrm{CN}-\mathrm{Ag}-\mathrm{CN}-$. Известно, что материалы с таким аномальным поведением (отрицательная линейная сжимаемость) весьма привлекательны для применения в датчиках давления, несжимаемых композитах [15]. Для цианида натрия линейная сжимаемость вдоль ковалентных связей $\mathrm{C}-\mathrm{N}$ цианид-анионов (вдоль оси $c$ ) меньше чем вдоль $a$ и $b$, однако она на порядок больше, чем для цианидов золота и серебра. Таким образом, упругая анизотропия для $\mathrm{NaCN}$ меньше чем для $\mathrm{AgCN}$ и $\mathrm{AuCN}$.

На рис. 3 приведены вычисленные зависимости от давления для длин связей $\mathrm{M}-\mathrm{N}$ и $\mathrm{M}-\mathrm{C}(\mathrm{M}=\mathrm{Ag}, \mathrm{Au}, \mathrm{Na})$ цианидов серебра, золота и натрия. Видно, что для $\mathrm{AgCN}$ и $\mathrm{AuCN}$ длины связей $\mathrm{M}-\mathrm{N}$ с ростом давления изменяются в значительно большей степени, чем длины связей $\mathrm{M}-\mathrm{C}$. При этом длина связи $\mathrm{Ag}-\mathrm{N}$ увеличивает- 

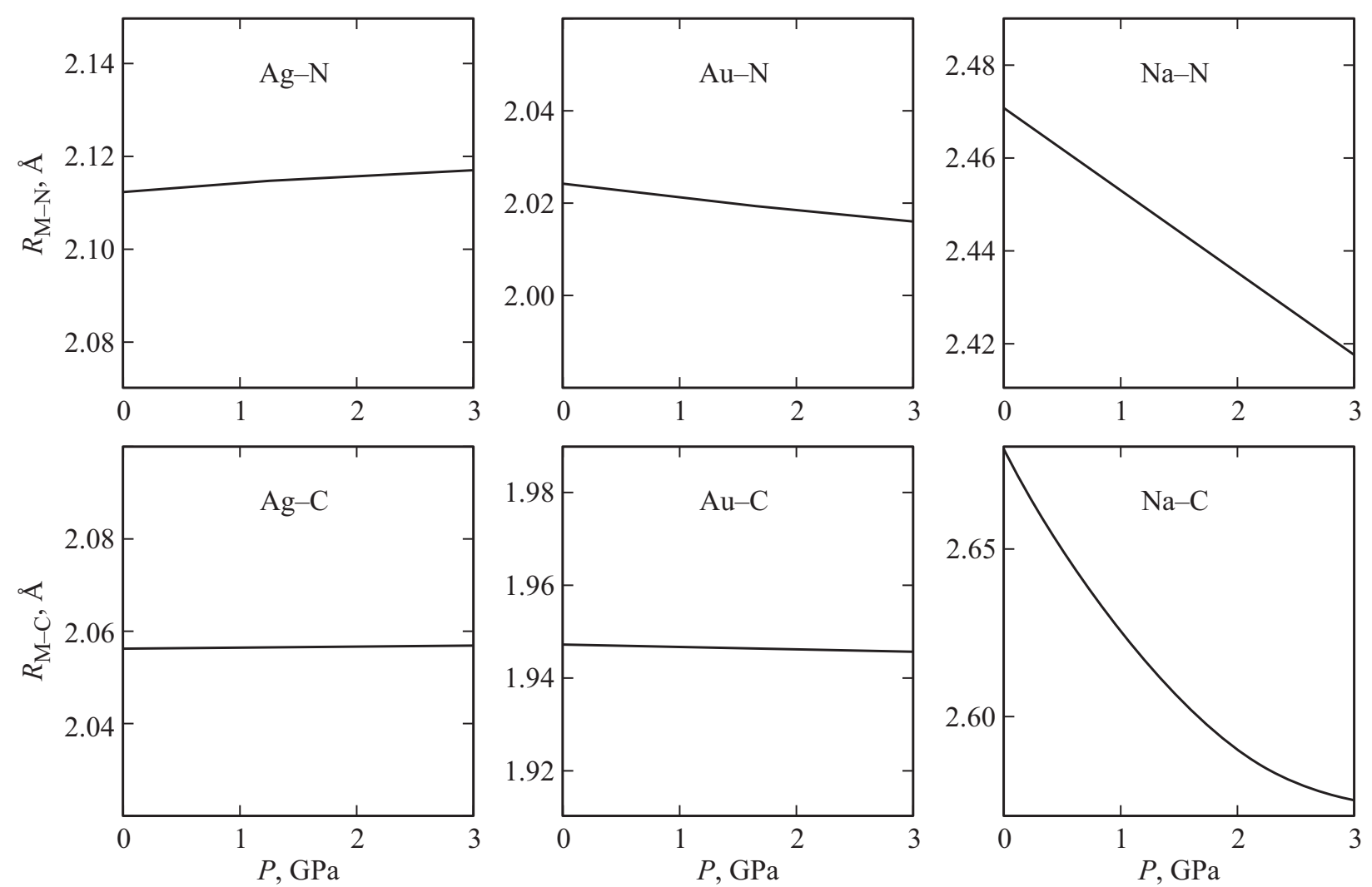

Рис. 3. Рассчитанные зависимости от давления для длин связей $\mathrm{M}-\mathrm{N}$ и $\mathrm{M}-\mathrm{C}(\mathrm{M}=\mathrm{Ag}, \mathrm{Au}, \mathrm{Na})$ цианидов металлов.
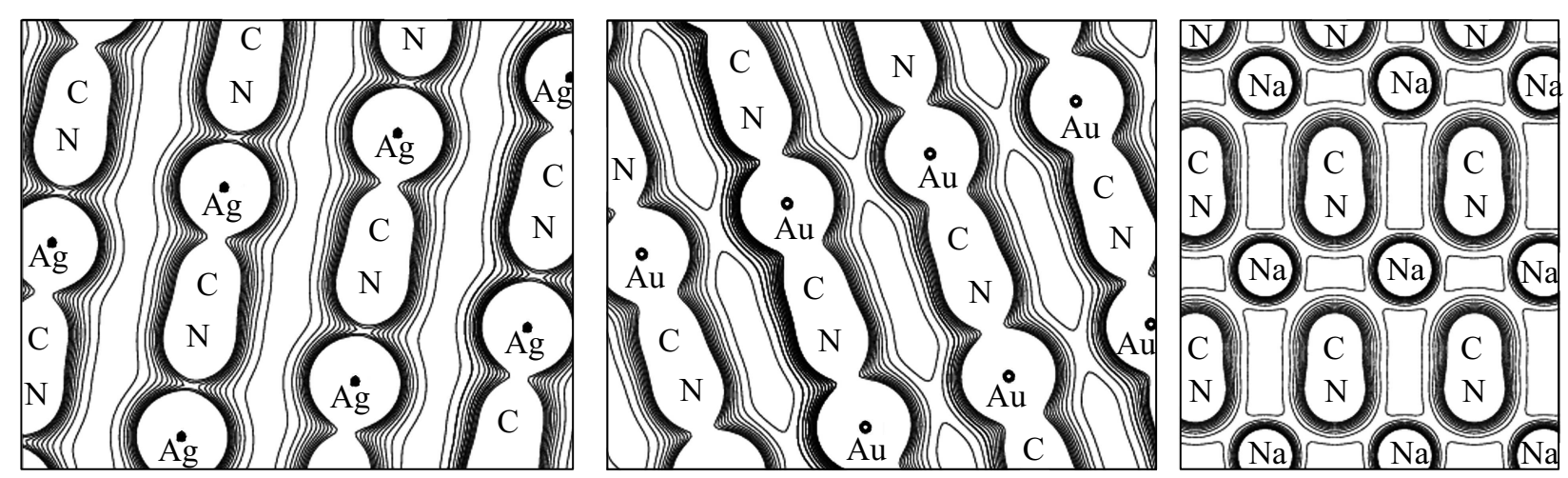

Рис. 4. Карты электронной плотности цианидов металлов.

ся под давлением (рис. 3). Таким образом, отрицательная линейная сжимаемость цианида серебра коррелирует со сжимаемостью его координационных связей $\mathrm{Ag}-\mathrm{N}$. Для цианида натрия давление вызывает значительно большее изменение длин связей $\mathrm{M}-\mathrm{N}$ и $\mathrm{M}-\mathrm{C}$, чем для $\mathrm{AgCN}$ и $\mathrm{AuCN}$. При этом межатомные расстояния $\mathrm{Na}-\mathrm{C}$ уменьшаются даже сильнее $(0.04 \AA / \mathrm{GPa})$, чем расстояния $\mathrm{Na}-\mathrm{N}(0.02 \AA / \mathrm{GPa})$. Длина связи $\mathrm{N}-\mathrm{C}$ для цианидов составляет $\sim 1.2 \AA$ и почти не меняется с ростом давления по сравнению со связями $\mathrm{M}-\mathrm{N}$.

На рис. 4 показаны распределения электронной плотности цианидов серебра, золота и натрия.
Видно, что в цианидах серебра и золота для цианогрупп и катионов металлов характерно наличие большого числа общих контуров. Интересно отметить наличие общего контура для катионов золота соседних цепочек $-\mathrm{Au}-\mathrm{CN}-\mathrm{Au}-\mathrm{CN}-$. B отличие от цианидов серебра и золота, для катионов и анионов цианида натрия имеет место только один общий контур. Далее в настоящей работе на основе QTAIM теории Бейдера был проведен топологический анализ электронной плотности для количественного исследования катион-анионных и катион-катионных взаимодействий в цианидах металлов. Такой QTAIM анализ позволяет установить наличие 


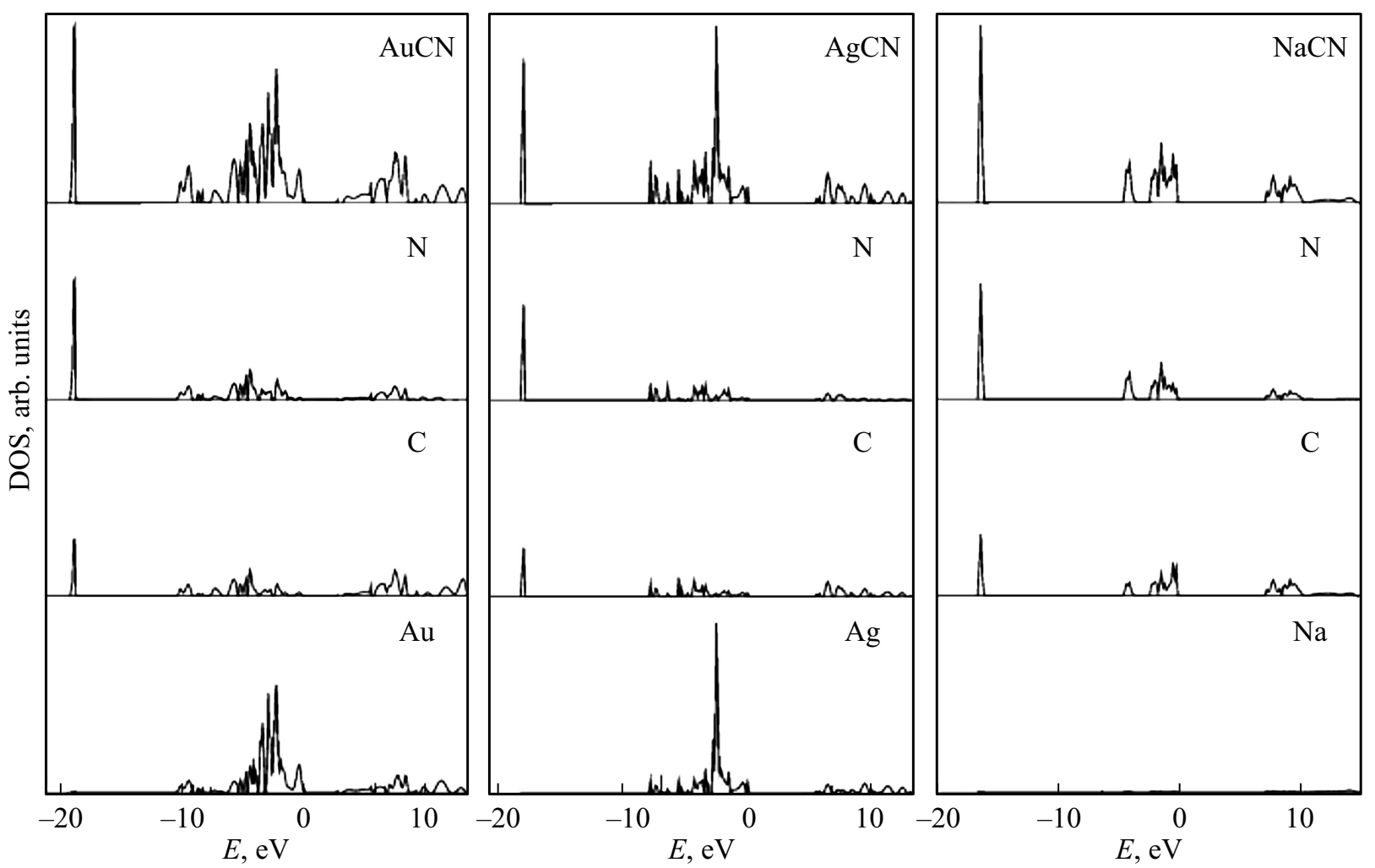

Рис. 5. Полные и парциальные плотности электронных состояний цианидов металлов.

связывающих взаимодействий, а также определить их природу и энергии [50-56]. В табл. 2 приведены значения электронной плотности $\rho_{c}$, лапласиана $\Delta \rho_{c}$ и плотности энергии $H_{c}$ в критических точках связей $\mathrm{M} \cdots \mathrm{M}, \mathrm{M}-\mathrm{C}$ и $\mathrm{M}-\mathrm{N}$, а также соответствующие энергии взаимодействий $E_{\text {int }}$.

Взаимодействия, характеризуемые положительными величинами как лапласиана $\Delta \rho_{c}$, так и плотности энергии $H_{c}$, относят к взаимодействиям типа закрытых оболочек (электростатические, дисперсионные) [50-53]. Из табл. 2 видно, что к таким взаимодействиям относятся связи $\mathrm{Na}-\mathrm{C}, \mathrm{Na}-\mathrm{N}$ и $\mathrm{Au} \cdots \mathrm{Au}$. Известно, что взаимодействия промежуточного типа (частично ковалент-

Таблица 2. Топологические параметры электронной плотности в критических точках катион-анионных и катионкатионных взаимодействий для цианидов металлов

\begin{tabular}{c|c|c|c|c|c}
\hline $\mathrm{MCN}$ & Связи $\mathrm{A}-\mathrm{B}$ & $\rho_{c}$, a.e. & $\Delta \rho_{c}$, a.e. & $H_{c}$, a.e. & $E_{\text {int }}, \mathrm{kJ} / \mathrm{mol}$ \\
\hline \multirow{3}{*}{$\mathrm{AgCN}$} & $\mathrm{Ag}-\mathrm{C}$ & 0.108 & 0.307 & -0.0408 & 208 \\
& $\mathrm{Ag}-\mathrm{N}$ & 0.084 & 0.400 & -0.0149 & 170 \\
\hline \multirow{3}{*}{$\mathrm{AuCN}$} & $\mathrm{Au}-\mathrm{C}$ & 0.152 & 0.357 & -0.0687 & 298 \\
& $\mathrm{Au}-\mathrm{N}$ & 0.115 & 0.516 & -0.0286 & 245 \\
& $\mathrm{Au} \cdots \mathrm{Au}$ & 0.014 & 0.051 & 0.0019 & 11.7 \\
\hline \multirow{3}{*}{$\mathrm{NaCN}$} & $\mathrm{Na}-\mathrm{C}$ & 0.012 & 0.058 & 0.0026 & 12.1 \\
& $\mathrm{Na}-\mathrm{N}$ & 0.016 & 0.083 & 0.0036 & 17.8
\end{tabular}

ные, слабые ковалентные), имеющие ковалентную компоненту, характеризуются отрицательными величинами $H_{c}$ при положительных значениях $\Delta \rho_{c}$ [51-53]. Таким образом, в отличие от цианида натрия, катион-анионные взаимодействия (связи $\mathrm{M}-\mathrm{C}$ и $\mathrm{M}-\mathrm{N}$ ) в цианидах золота и серебра имеют частично ковалентную природу. Взаимодействия между катионами (металлофильные) имеют место только для цианида золота (аурофильные взаимодействия $\mathrm{Au} \cdots \mathrm{Au}$ с энергиями $12 \mathrm{~kJ} / \mathrm{mol})$. Из табл. 2 видно, что энергии катион-анионных взаимодействий в цианидах золота и серебра на порядок больше, чем для цианида натрия. При этом для цианида золота энергии связей $\mathrm{M}-\mathrm{C}$ и $\mathrm{M}-\mathrm{N}$ наиболее значительны.

На рис. 5 изображены полные плотности электронных состояний $\mathrm{MCN}(\mathrm{M}=\mathrm{Au}, \mathrm{Ag}, \mathrm{Na})$ и их парциальные вклады от атомов M, C и N. Энергия самых верхних занятых состояний положена равной нулю.

Видно, что верхние валентные состояния цианидов золота и серебра формируются в основном состояниями катионов (Au и $\mathrm{Ag}$ ) при энергиях от нуля до -10.1 и $-7.7 \mathrm{eV}$ соответственно. Наоборот, верхние валентные состояния цианида натрия отвечают гибридизованным состояниям углерода и азота при энергиях от нуля до $-4.4 \mathrm{eV}$. Общим для валентной области цианидов золота, серебра и натрия является пик анионных состояний при энергиях 18.7, 17.7 и $16.3 \mathrm{eV}$ соответственно.

Известно, что в ряде случаев градиентные функционалы, в частности функционал PBE [41], приводят к 

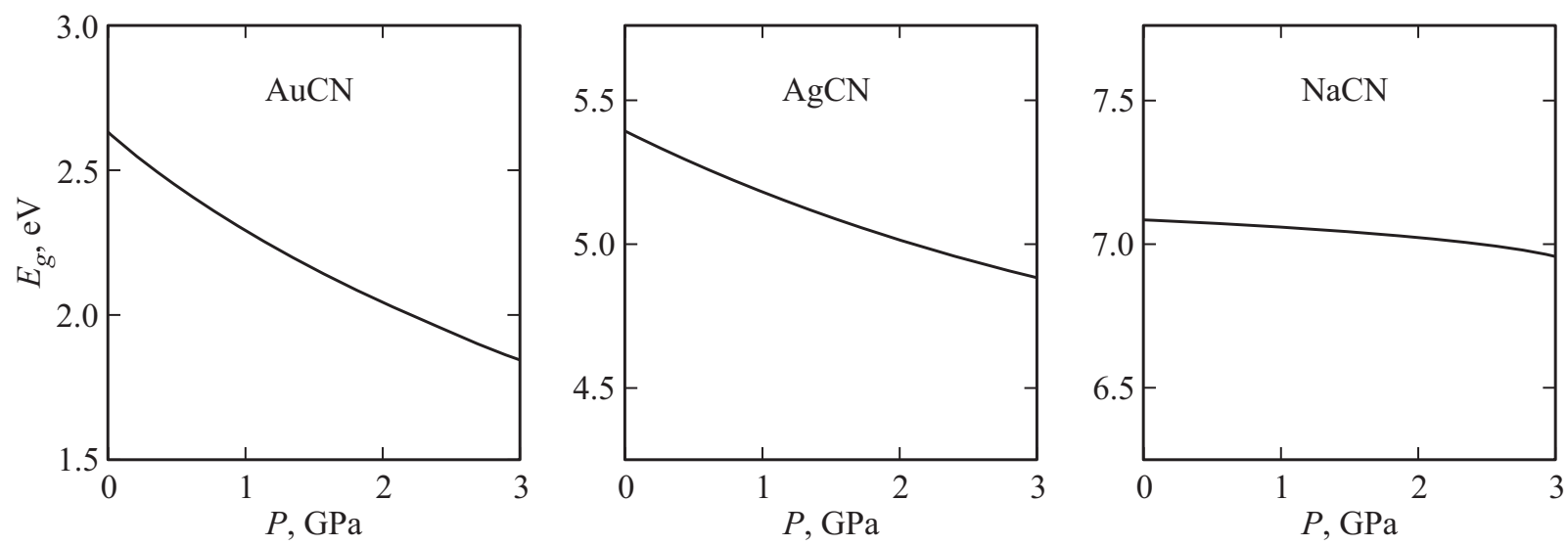

Рис. 6. Зависимости от давления для ширин запрещенных зон цианидов металлов.

недооценке значений ширины запрещенной зоны. В этом случае лучшего согласия с экспериментом позволяют добиться так называемые гибридные функционалы. Ширина запрещенной зоны, вычисленная нами с помощью гибридного функционала B3LYP [42], для цианида золота составляет $2.64 \mathrm{eV}$, что хорошо согласуется с ее экспериментальным значением при нормальных условиях $(2.35 \mathrm{eV}[25])$. Для цианидов серебра и натрия вычисленная в настоящей работе ширина запрещенной зоны равна 5.39 и $7.08 \mathrm{eV}$ соответственно. Таким образом, ширина запрещенной зоны цианида золота меньше, чем для цианидов серебра и натрия. При этом наибольшая $E_{g}$ отвечает $\mathrm{NaCN}$.

Ширины запрещенных зон цианидов металлов при увеличении внешнего давления уменьшаются (рис. 6).

Из рис. 6 видно, что ширины запрещенных зон цианидов золота и серебра с ростом давления уменьшаются значительно больше, чем для цианида натрия. Так, в линейной аппроксимации $E_{g}$ для $\mathrm{AuCN}$ уменьшается как $0.261 \mathrm{eV} / \mathrm{GPa}$, что хорошо согласуется с экспериментальной величиной $d E_{g} / d P=0.25 \mathrm{eV} / \mathrm{GPa}$ [25]. Таким образом, согласно нашим расчетам при давлении $10.1 \mathrm{GPa}$ должна произойти металлизация цианида золота $\left(E_{g}=0 \mathrm{eV}\right)$, что соответствует экспериментальным измерениям [25]. Для цианида серебра скорость изменения ширины запрещенной зоны с давлением $\left(d E_{g} / d P\right)$ составляет $0.172 \mathrm{eV} / \mathrm{GPa}$ и металлизация для $\mathrm{AgCN}$ должна произойти при $31.3 \mathrm{GPa}$. С другой стороны, $d E_{g} / d P=0.042 \mathrm{eV} / \mathrm{GPa}$ для $\mathrm{NaCN}$.

\section{4. Заключение}

C помощью программного пакета CRYSTAL вычислены параметры решетки, длины и энергии связей, зарядовые распределения и топологические параметры электронной плотности, полные и парциальные плотности электронных состояний, ширины запрещенных зон цианидов металлов $\mathrm{MCN}(\mathrm{M}=\mathrm{Ag}, \mathrm{Au}, \mathrm{Na})$. В результате проведенных расчетов изучены структурные и электрон- ные свойства цианидов металлов, а также влияние на них внешнего давления.

Вычисленные величины постоянных решетки согласуются с известными экспериментальными данными (отличия менее 2\%). Выявлено, что цианид серебра имеет наименьший модуль объемного сжатия. Линейная сжимаемость цианидов металлов сильно анизотропна, в особенности для $\mathrm{AgCN}$ и $\mathrm{AuCN}$. Линейный модуль вдоль цепочек $-\mathrm{Au}-\mathrm{CN}-\mathrm{Au}-\mathrm{CN}-$ сопоставим с линейными модулями алмаза и нитрида бора. Показано, что давление вызывает увеличение линейных размеров $\mathrm{AgCN}$ вдоль цепочек $-\mathrm{Ag}-\mathrm{CN}-\mathrm{Ag}-\mathrm{CN}-$. Таким образом, для $\mathrm{AgCN}$ характерна отрицательная линейная сжимаемость. Для цианида натрия линейная сжимаемость вдоль цианид-анионов меньше чем в других направлениях. Длина связи $\mathrm{Ag}-\mathrm{N}$ увеличивается под давлением и, таким образом, отрицательная линейная сжимаемость цианида серебра коррелирует со сжимаемостью его координационных связей $\mathrm{Ag}-\mathrm{N}$. Установлено, что для цианида натрия давление вызывает значительно большее изменение длин связей $\mathrm{M}-\mathrm{N}$ и $\mathrm{M}-\mathrm{C}$, чем для $\mathrm{AgCN}$ и $\mathrm{AuCN}$.

Выявлено, что в отличие от цианида натрия, катионанионные взаимодействия (связи $\mathrm{M}-\mathrm{C}$ и $\mathrm{M}-\mathrm{N}$ ) в цианидах золота и серебра имеют частично ковалентную природу. Металлофильные взаимодействия имеют место только для цианида золота (аурофильные взаимодействия $\mathrm{Au} \cdots \mathrm{Au})$. Показано, что энергии катионанионных взаимодействий в цианидах золота и серебра на порядок больше, чем для цианида натрия. При этом для цианида золота энергии связей $\mathrm{M}-\mathrm{C}$ и $\mathrm{M}-\mathrm{N}$ наиболее значительны.

Показано, что, в отличие от цианида натрия, верхние валентные состояния цианидов золота и серебра формируются в основном состояниями катионов. Вычисленная ширина запрещенной зоны цианида золота хорошо согласуется с ее экспериментальным значением. Установлено, что ширина запрещенной зоны цианида золота меньше, чем для цианидов серебра и натрия. Ширины запрещенных зон цианидов золота и серебра с ростом 
внешнего давления уменьшаются значительно больше, чем для цианида натрия. Для цианида золота скорость изменения ширины запрещенной зоны с давлением $\left(d E_{g} / d P\right)$ хорошо согласуется с экспериментальными данными. При достаточно больших давлениях возможна металлизация цианидов золота и серебра.

\section{Конфликт интересов}

Авторы заявляют об отсутствии конфликта интересов.

\section{Список литературы}

[1] G. A. Bowmaker, B.J. Kennedy, J.C. Reid. Inorg. Chem. 37, 3968 (1998).

[2] D. Fontaine. Comptes Rendus Hebdomadaires des Seances de I'Academie des Sciences B 281, 443 (1975).

[3] S.J. Hibble, J.B. Wood, E.J. Bilbe, A.H. Pohl, M.G. Tucker, A.C. Hannon, A.M. Chippindale. Z. Kristallogr. 225, 457 (2010).

[4] М.Ю. Петрушина, С.В. Коренев, Е.С. Дедова, А.И. Губанов. Журн. структур. химии. 61, 1749 (2020).

[5] K. Takenaka. Sci. Technol. Adv. Mater. 13, 013001 (2012).

[6] W. Miller, C. Smith, D. Mackenzie, K. Evans. J. Mater. Sci. 44, 5441 (2009).

[7] D. Das, T. Jacobs, L.J. Barbour. Nature Mater 9, 36 (2010).

[8] R.W. Munn. J. Phys. C 5, 535 (1972).

[9] A.D. Fortes, E. Suard, K.S. Knight. Science 331, 742 (2011).

[10] S. Hodgson, J. Adamson, S. Hunt, M. Cliffe, A.B. Cairns, A.L. Goodwin. Chem. Commun. 50, 5264 (2014).

[11] K. Dolabdjian, A. Kobald, C.P. Romao, H. Meyer. Dalton Trans. 47, 10249 (2018).

[12] L. Wang, C. Wang, H. Luo, Y. Sun. J. Phys. Chem. C 121, 333 (2017).

[13] M.Yu. Seyidov, R.A. Suleymanov. J. Appl. Phys. 108, 063540 (2010).

[14] C.P. Romao, S.P. Donegan, J.W. Zwanziger, M.A. White. Phys. Chem. Chem. Phys. 18, 30652 (2016).

[15] A.B. Cairns, A.L. Goodwin. Phys. Chem. Chem. Phys. 17, 20449 (2015)

[16] W. Cai, A. Katrusiak. Nature Commun. 5, 4337 (2014).

[17] P. Serra-Crespo, A. Dikhtiarenko, E. Stavitski, J. Juan-Alcaniz, F. Kapteijn, F.-X. Coudert, J. Gascon. Cryst. Eng.Commun. 17, 276 (2015).

[18] S. Duyker, V. Peterson, G. Kearley, A. Studer, C. Kepert. Nature Chem. 8, 270 (2016).

[19] H. Wang, M. Feng, Y. Wang, Z. Gu. Sci. Rep. 6, 26015 (2016).

[20] D.V. Korabel'nikov, Yu.N. Zhuravlev. Phys. Chem. Chem. Phys. 18, 33126 (2016).

[21] D.V. Korabel'nikov, Yu.N. Zhuravlev. J. Phys. Chem. A 121, 6481 (2017).

[22] S. Sobczak, A. Porolniczak, W. Cai, A. Gadysiak, V.I. Nikolayenko, D. Castell, L. Barbour, A. Katrusiak. Chem. Commun. 56, 4324 (2020).

[23] A.Y. Liu, M.L. Cohen. Science 245, 841 (1989).

[24] Q. Fan, C. Chai, Q. Wei, Y. Yang. Materials 9, 427 (2016).

[25] R.S. Bradley, D.C. Munro, P.S. Spencer. Phys. Status Solidi 36, K51 (1969)

[26] A.D. Becke. J. Chem. Phys. 140, 18A301 (2014).

[27] D.C. Sorescu, B.M. Rice. J. Phys. Chem. C 114, 6734 (2010).
[28] S. Appalakondaiah, G. Vaitheeswaran, S. Lebegue. J. Chem. Phys. 138, 184705 (2013).

[29] S. Hunter, P. Coster, A. Davidson, D. Millar, S. Parker, W. Marshall, R. Smith, C. Morrison, C. Pulham. J. Phys. Chem. C 119, 2322 (2015).

[30] I.A. Fedorov, Yu.N. Zhuravlev. Chem. Phys. 436, 1 (2014).

[31] D.V. Korabel'nikov, Yu.N. Zhuravlev. J. Phys. Chem. Solids. 87, 38 (2015).

[32] Д.В. Корабельников, Ю.Н. Журавлев. ФТТ 59, 248 (2017).

[33] I.A. Fedorov. Comput. Mater. Sci. 139, 252 (2017).

[34] A.R. Oganov, C.W. Glass. J. Phys.: Condens. Matter. 20, 064210 (2008).

[35] A.G. Kvashnin, Z. Allahyari, A.R. Oganov. J. Appl. Phys. 126, 040901 (2019).

[36] R. Dovesi, A. Erba, R. Orlando, C.M. Zicovich-Wilson, B. Civalleri, L. Maschio, M. Rerat, S. Casassa, J. Baima, S. Salustro, B. Kirtman. WIREs Comput. Mol. Sci. 8, e1360 (2018).

[37] E. Apra, E. Stefanovich, R. Dovesi, C. Roetti. Chem. Phys. Lett. 186, 329 (1991).

[38] P.J. Hay, W.R. Wadt. J. Chem. Phys. 82, 299 (1985)

[39] R. Dovesi, C. Roetti, C. Fava, M. Prencipe, V.R. Saunders. Chem. Phys. 156, 11 (1991)

[40] C. Gatti, V.R. Saunders, C. Roett. J. Chem. Phys. 101, 10686 (1994).

[41] J.P. Perdew, K. Burke, M. Ernzerhof. Phys. Rev. Lett. 77, 3865 (1996).

[42] A.D. Becke. J. Chem. Phys. 98, 5648 (1993).

[43] S. Grimme, J. Antony, S. Ehrlich, H. Krieg. J. Chem. Phys. 132, 154104 (2010).

[44] S. Grimme, S. Ehrlich, L. Goerigk. J. Comput. Chem. 32, 1456 (2011).

[45] C.G. Broyden. J. Appl. Math. 6, 222 (1970).

[46] D.V. Korabel'nikov, Yu.N. Zhuravlev. RSC Advances 10, 42204 (2020).

[47] R.F.W. Bader. Chem. Rev. 91, 893 (1991).

[48] A.O. Borissova, A.A. Korlyukov, M.Y. Antipind, K.A. Lyssenko. J. Phys. Chem. A 112, 11519 (2008).

[49] C. Gatti, S. Casassa. TOPOND14 User's Manual. CNR-ISTM Milano, Milano (2014).

[50] R.F.W. Bader. Atoms in Molecules - A Quantum Theory. Oxford University Press, Oxford (1990).

[51] D. Cremer, E. Kraka. Angew. Chem. Int. Ed. 23, 627 (1984).

[52] E. Espinosa, I. Alkorta, J. Elguero, E. Molins. J. Chem. Phys. 117, 5529 (2002).

[53] C. Gatti. Z. Kristallogr. 220, 399 (2005).

[54] E. Espinosa, E. Molins, C. Lecomte. Chem. Phys. Lett. 285, 170 (1998).

[55] E.A. Zhurova, A.I. Stash, V.G. Tsirelson, V.V. Zhurov, E.V. Bartashevich, V.A. Potemkin, A.A. Pinkerton. J. Am. Chem. Soc. 128, 14728 (2006).

[56] D.V. Korabel'nikov, Yu.N. Zhuravlev. RSC Advances. 9, 12020 (2019).

Редактор Т.Н. Василевская 